\title{
Outcome after Lacerations of the Superficial and Deep Digital Flexor Tendons, Suspensory Ligament and/or Distal Sesamoidean Ligaments in 106 Horses
}

\author{
Mireia Jordana ${ }^{1}$, DVM, Hans Wilderjans ${ }^{2}$, DVM, Diplomate ECVS, Jane Boswell ${ }^{3}$, MA, Vet MB, Cert VA, \\ Diplomate ECVS, Jeroen Dewulf ${ }^{4}$, DVM, MSC, PhD, Diplomate ECVPH, Roger K. W. Smith ${ }^{5}$, MA, Vet MB, \\ PhD, DEO, Diplomate ECVS, and Ann Martens ${ }^{1}$, DVM, PhD, Diplomate ECVS \\ ${ }^{1}$ Department of Surgery and Anaesthesiology of Domestic Animals, Faculty of Veterinary Medicine, Ghent University, Merelbeke, Belgium, \\ ${ }^{2}$ Dierenkliniek De Bosdreef-Dierenkliniek De Morette, Moerbeke-Waas, Belgium, ${ }^{3}$ Liphook Equine Hospital, Hampshire, UK, ${ }^{4}$ Department of \\ Reproduction, Obstetrics and Herd Health, Veterinary Epidemiology Unit, Faculty of Veterinary Medicine, Ghent University, Merelbeke, Belgium and \\ ${ }^{5}$ Department of Veterinary Clinical Sciences, Royal Veterinary College, North Mymms, UK
}

\author{
Corresponding Author \\ Ann Martens, DVM, PhD, Diplomate ECVS, \\ Department of Surgery and \\ Anaesthesiology of Domestic Animals, \\ Faculty of Veterinary Medicine, Ghent \\ University, Salisburylaan 133, 9820 \\ Merelbeke, Belgium \\ E-mail: ann.martens@UGent.be
}

Submitted January 2010

Accepted December 2010

DOI:10.1111/j.1532-950X.2011.00814.x

\begin{abstract}
Objective: To report outcome after the surgical treatment of lacerations of the superficial digital flexor tendon (SDFT), deep digital flexor tendon (DDFT), suspensory ligament (SL), and/or distal sesamoidean ligaments (DSL) in horses.

Study Design: Case series.

Animals: Horses $(\mathrm{n}=106)$ with lacerations of the SDFT, DDFT, SL, and/or DSL. Methods: Medical records (1988-2002) were reviewed for signalment, limb and tendon/ligament involvement (location and extent of injury, tendon sheath involvement), method of repair, and outcome.

Results: The median age of horses was 7 years and the follow-up time ranged from 1 to 10 years. Fifty-five percent of horses returned to their previous level of performance, $27 \%$ to a lower level, and $18 \%$ were euthanatized. Multivariate statistical analysis demonstrated that the number of structures transected had the most significant influence on outcome. No significant association was detected between outcome and tendon sheath involvement, tendon suturing, casting, or limb affected. Fetlock hyperextension was the most significant complication.

Conclusions: A high survival rate can be expected after SDFT, DDFT, SL, and/or DSL lacerations in horses, but only $55 \%$ of affected horses returned to their previous activity level. The number of structures affected was the major factor determining whether horses returned to an equal level of performance.
\end{abstract}

Injuries to the distal aspect of the limbs are common in horses and frequently involve injury to the extensor and flexor tendons, suspensory ligament (SL), or distal sesamoidean ligaments (DSL). Concurrent injury to bone, synovial cavities, and neurovascular structures often occurs. The prognosis for return to the previous level of work is influenced by factors including degree of skin damage, which tendons or ligaments are injured, and whether a synovial structure is involved. ${ }^{1}$ Flexor tendon lacerations are often associated with minimal skin damage even with complete tendon transection. ${ }^{2}$ Because of the importance of the flexor tendons, SL and DSL in load bearing, characteristic alterations in limb conformation and gait are observed when these structures are disrupted.

Transection of the superficial digital flexor tendon (SDFT) results in hyperextension of the metacarpo/metatarsophalangeal (MCP/MTP) joint when the limb is loaded. ${ }^{3}$ When the SDFT and deep digital flexor tendon (DDFT) are lacerated, the MCP/MTP joint may hyper- extend and the toe lifts from the ground. ${ }^{3}$ If the SDFT, DDFT, and SL are transected, there is complete loss of support of the $\mathrm{MCP} / \mathrm{MTP}$ joint with hyperextension until the joint contacts the ground. ${ }^{3}$ If horses are not completely weight bearing, it may be difficult to observe these clinical signs of altered limb support.

Surgical treatment is generally recommended; however, the value of tenorrhaphy and/or cast immobilization has been questioned. ${ }^{4,5}$ Return to the original level of work has been reported in $18 \%{ }^{4}$ and $54 \%{ }^{5}$ of horses after flexor tendon lacerations; however, the sample sizes in these studies were relatively small, $22^{4}$ and $50^{5}$ horses, respectively. Because laceration results in varying involvement and severity of injury of these structures, the identification of specific prognostic factors is challenging. Thus, our purpose was to: (1) assess outcome after the surgical treatment of lacerations of the SDFT, DDFT, SL, or DSL in a larger group of horses and (2) determine whether the type and location of injury, type of treatment, and development of 
postsurgical complications had an influence on outcome, especially return to the previous activity level.

\section{MATERIALS AND METHODS}

\section{Horses}

Medical records (1988-2002) of horses that had surgical treatment for partial or complete lacerations of the SDFT, DDFT, SL, and/or DSL below the carpus or tarsus at 4 referral clinics were reviewed. Horses evaluated for tendon or ligament lacerations but not treated because of financial constraints were excluded. Horses euthanatized during surgery because of an extremely poor prognosis were included.

Data retrieved were signalment, limb involvement, number and type of tendons and ligaments affected (SDFT, DDFT, SL, and/or DSL), degree of tendon laceration (partial, total), laceration location (proximal, mid, distal third of the metacarpal or metatarsal regions or at the level of the pastern), and tendon sheath (digital, tarsal, or carpal) involvement. Cases were classified according to the number of structures affected $(1,2$, or 3 tendons or ligaments, partially or completely lacerated) and the severity of laceration: group $1=$ horses with partial transection of 1 or more tendons or ligaments (SDFT, DDFT, SL, and/or DSL) and group $2=$ horses with complete transection of 1 or more tendons or ligaments (SDFT, DDFT, SL, and/or DSL). Additionally, the type of treatment, in particular, whether the tendon or ligament was sutured (no implants were used), whether a bandage or cast was used, and duration of cast immobilization, was retrieved.

\section{Outcome}

Long-term follow-up information was obtained by a telephone questionnaire of owners and/or referring veterinary surgeons. Whenever possible, horses were reexamined at the referral clinic. The presence of lameness and other complications was recorded. Development of hyperextension of the MCP/MTP joint compared with the opposite limb after rehabilitation was assessed by the owner/trainer at home or by the veterinary surgeon whenever possible. The horse's level of work before and after injury was ascertained. Outcome was defined as "equal performance" when the horse returned to its original or intended level of use or "lower performance" when the horse did not return to that level, including those used for breeding or left in pasture. Horses that were euthanatized during or after surgery for reasons directly or indirectly related to the tendon laceration were defined as "euthanasia".

\section{Statistical Analysis}

For statistical analysis, outcome was classified as "successful" when an equal performance was obtained or "unsuccessful" when performance was less than expected or the horse was euthanatized. Associations between clinical findings, treatment, postsurgical complications, and outcome were analyzed using logistic regression (SPSS 11.0, SPSS
Inc., Chicago, IL). Univariate analysis was performed initially for all independent variables. Subsequently, all variables with a $P$-value $\leq .2$ were selected for the multivariate analysis. Spearman's $\rho$ correlation between all independent variables was calculated and if the correlation was $>0.6$ then only the most significant value was retained. The multivariate model was built using a manual stepwise backward procedure. ${ }^{6}$

A Pearson $\chi^{2}$ test (SPSS 11.0, SPSS Inc.) was used to evaluate the association between the presence of a partial or a complete tendon or ligament laceration and whether tenorrhaphy was performed or casting was used.

\section{RESULTS}

\section{Horses}

One hundred and six horses (median age, 7 years; range, 8.5 months to 23 years; 50 males [41 geldings, 9 stallions], 56 females) were treated. The distribution of horses between clinics was University of Ghent, 37; Dierenkliniek De Bosdreef-Dierenkliniek De Morette, 29; Liphook Equine Hospital, 20; and Royal Veterinary College, 20. There were 46 Warmbloods, 24 Thoroughbreds, 11 ponies, 8 Standardbreds, 6 Arabians, 2 Quarter horses, 1 Irish cross-bred, and 8 horses whose breed was not recorded. Time between injury and referral was not consistently recorded.

\section{Lesion Distribution (Table 1)}

Lacerations involved the forelimb in 39 horses $(37 \%)$ and the hind limb in $67(63 \%)$. Radiographic examination of horses with suspected bone involvement identified 3 with fractures of the 2 nd or the 4th metatarsal bone. Synoviocentesis was performed when penetration of a synovial cavity was suspected. In most horses, the diagnosis of tendon or ligament laceration was obvious on physical examination but the degree of laceration and the number and type of structures involved were often only possible to determine during surgery.

One tendon was partially or totally lacerated in 48 horses, 2 tendons in 45 horses, and 3 structures in 13 horses. The most common types of lacerations in group 1 horses $(n=60[57 \%])$ were partial transections of the SDFT $(27 ; 45 \%)$, partial lacerations of both SDFT and DDFT $(13 ; 22 \%)$, and of the DDFT only $(10 ; 17 \%)$. The degree of partial tendon laceration varied from minor to substantial but precise information on the percentage of tendon involvement could not be obtained from all records. Group 2 horses $(46 ; 43 \%)$ had at least total laceration of the SDFT or DDFT. Total transection of the SDFT with a partial laceration of the DDFT was the most common injury $(17 ; 37 \%)$. Ten horses $(22 \%)$ had complete transection of both SDFT and DDFT and 6 horses (13\%) had complete transection of both SDFT and DDFT and partial transection of the SL or DSL. 
Table 1 Summary of Injury Characteristics by Group for 106 Horses with Lacerations of the Digital Flexor Tendons, Suspensory Ligament, and/or Distal Sesamoidean Ligaments

\begin{tabular}{|c|c|c|c|c|c|c|c|c|c|c|c|c|c|}
\hline & \multicolumn{3}{|c|}{$\begin{array}{c}\text { Structures } \\
\text { Affected }(n=106)\end{array}$} & \multirow[b]{2}{*}{$\begin{array}{c}\text { Total } \\
(n=106)\end{array}$} & \multicolumn{4}{|c|}{$\begin{array}{l}\text { Limb Region } \\
\qquad(\mathrm{n}=106)\end{array}$} & \multirow{2}{*}{$\begin{array}{c}\text { Open } \\
\text { Tendon Sheath } \\
n=61(58 \%)\end{array}$} & \multirow{2}{*}{$\begin{array}{c}\text { Sutured } \\
\text { Tendon } \\
\mathrm{n}=58(55 \%)^{*}\end{array}$} & \multicolumn{2}{|c|}{ Casting $(n=104)^{*}$} & \multirow{2}{*}{$\begin{array}{c}\text { Fetlock } \\
\text { Hyperextension } \\
(\mathrm{n}=15) \dagger\end{array}$} \\
\hline & SDFT & DDFT & SL/DSL & & $\operatorname{Pr}$ & M & $D$ & $\mathrm{~Pa}$ & & & $\begin{array}{c}\text { Number of } \\
\text { Horses }(n=84)\end{array}$ & $\begin{array}{l}\text { Period (Weeks) } \\
\text { Median [Range] }\end{array}$ & \\
\hline \multirow[t]{7}{*}{ Group 1} & & & & $60(57 \%)$ & & & & & 36 & 24 & $\begin{array}{l}41(68 \% \text { of } \\
\text { Group 1) }\end{array}$ & $4.7[1-12]$ & 1 \\
\hline & $P$ & $x$ & $x$ & 27 & 1 & 8 & 13 & 5 & 13 & 9 & 18 & $4.8[2-12] \ddagger$ & \\
\hline & $P$ & $P$ & $x$ & 13 & 1 & 1 & 4 & 7 & 9 & 9 & 10 & $6.2[1.5-12]$ & \\
\hline & $x$ & $P$ & $x$ & 10 & & & & 10 & 9 & 2 & 7 & 4.3 [3-7] & 1 \\
\hline & $x$ & $x$ & $P$ & 7 & & 1 & 5 & 1 & 2 & 3 & 4 & 1.75 [1-2] & \\
\hline & $P$ & $P$ & $P$ & 2 & & & 1 & 1 & 2 & 1 & 1 & 2 & \\
\hline & $P$ & $x$ & $P$ & 1 & & & 1 & & 1 & & 1 & 6 & \\
\hline \multirow[t]{12}{*}{ Group 2} & & & & $46(43 \%)$ & & & & & 25 & $34 *$ & $\begin{array}{l}43(98 \% \text { of } \\
\text { Group } 2)^{*}\end{array}$ & $7[0.5-14]$ & 14 \\
\hline & $\mathrm{T}$ & $P$ & $x$ & 17 & 3 & 8 & 4 & 2 & 7 & $12^{*}$ & $15^{*}$ & $6.7[0.5-14]$ & 3 \\
\hline & $\mathrm{T}$ & $\mathrm{T}$ & $x$ & 10 & 1 & 5 & 4 & & 5 & 9 & 10 & 8.5 [4-10] & 4 \\
\hline & $\mathrm{T}$ & $\mathrm{T}$ & $P$ & 6 & 2 & 1 & 2 & 1 & 4 & 6 & 6 & $7[1-11]$ & 4 \\
\hline & $\mathrm{T}$ & $x$ & $x$ & 4 & 1 & & 3 & & 3 & 2 & 4 & 6 [4-8] & \\
\hline & $\mathrm{T}$ & $P$ & $P$ & 2 & 1 & & & 1 & & 2 & 2 & $6 \ddagger$ & 1 \\
\hline & $\mathrm{T}$ & $\mathrm{T}$ & $\mathrm{T}$ & 2 & & & & 2 & 2 & 1 & 2 & 6 [4-8] & 1 \\
\hline & $\mathrm{T}$ & $x$ & $P$ & 1 & & 1 & & & & 1 & 1 & 12 & 1 \\
\hline & $P$ & $\mathrm{~T}$ & $P$ & 1 & & & & 1 & 1 & & 1 & 2 & \\
\hline & $P$ & $\mathrm{~T}$ & $x$ & 1 & & & & 1 & 1 & & & & \\
\hline & $x$ & $\mathrm{~T}$ & $\mathrm{~T}$ & 1 & & & & 1 & 1 & & 1 & 2 & \\
\hline & $x$ & $\mathrm{~T}$ & $x$ & 1 & & & & 1 & 1 & 1 & 1 & 6 & \\
\hline
\end{tabular}

Group 1, horses with partial transection of 1 or more tendons or ligaments (SDFT, DDFT, SL, and DSL).

Group 2, horses with complete transection of 1 or more tendons or ligaments (SDFT, DDFT, SL, and DSL).

* In 2 cases, suturing or cast application was not recorded.

$\dagger$ In 11 horses, the information on fetlock hyperextension was not available.

$\ddagger$ In 2 horses, the information for casting period was not available.

SDFT, superficial digital flexor tendon; DDFT, deep digital flexor tendon; $S$, suspensory ligament; DSL, distal sesamoidean ligaments; $\mathrm{P}$, partial transection of a tendon or a ligament; $\mathrm{T}$, total transection of a tendon or a ligament; $\mathrm{x}$, tendon or ligament not affected; Pr, proximal third of metacarpus/metatarsus; M, middle third of metacarpus/metatarsus; D, distal third of metacarpus/metatarsus; Pa, pastern.

\section{Treatment}

Similar surgical procedures were performed in all 4 clinics and except for 1 horse with atrial fibrillation treated using standing sedation, all other horses were anesthetized. Recovery from anesthesia was assisted with a head and tail rope in only 1 clinic. In most horses, the original skin wound was extended proximally and distally to allow thorough wound debridement and exposure of the lacerated tendon and ligament ends. In penetrating lacerations $(58 \%)$, synovial cavities were copiously lavaged with either through and through needle lavage or under tenoscopic control and local antibiotics were always administered (intrasynovial or by intravenous regional limb perfusion). In 1 horse, an additional lavage was required for the treatment of infection that persisted after the first treatment and in another horse 2 additional intrathecal lavages were needed.

Tendon ends were debrided but tenorrhaphy was only performed when apposition could be achieved. Tenorrhaphy was performed significantly more frequently in horses with complete tendon transection (group 2) than in horses with partial lacerations (group 1) (77\% versus $40 \%$, $P<.001)$. Suture patterns and material used were variable; however, a 3-loop pulley or a double-locking loop pattern with large-diameter polydioxanone was most common. When tenorrhaphy was not performed, the tendon healed by second intention. Overlying soft tissue and skin were closed as much as possible. All horses were administered systemic broad-spectrum antimicrobials and antiinflammatory drugs with dose and dosing intervals that varied by surgeon.

Immobilization with a lower limb cast was used in 84 of 104 horses $(79 \%, 2$ not recorded) for $0.5-14$ weeks (mean $\pm \mathrm{SD}, 6 \pm 3$ weeks). The wound was protected with a bandage in the other horses. Cast immobilization was used in a significantly higher proportion of group 2 horses $(98 \%)$ than group $1(68 \%, P<.001$; Table 1$)$. After cast removal, limbs were supported by simple or double-layer bandages. Corrective shoeing with heel extensions to prevent toe lifting was used when indicated.

\section{Outcome (Table 2)}

Follow-up information was obtained for all horses after at least 1 year (range, $1-10$ years); 58 (55\%) had a successful outcome and returned to an equal level of performance and 48 had an unsuccessful outcome (29 [27\%] returning to a lower level of performance and 19 [18\%] were euthanatized for reasons related to the tendon injury [ 3 perioperatively 
Table 2 Summary of Outcome Results by Injury Characteristics in 106 Horses with Lacerations of the Digital Flexor Tendons, Suspensory Ligament, and/or Distal Sesamoidean Ligaments

\begin{tabular}{|c|c|c|c|}
\hline & \multicolumn{3}{|c|}{ Outcome } \\
\hline & $\begin{array}{c}\text { Equal } \\
\text { Performance }\end{array}$ & $\begin{array}{c}\text { Lower } \\
\text { Performance }\end{array}$ & Euthanasia \\
\hline Number of horses $(n=106)$ & $58(55 \%)$ & $29(27 \%)$ & $19(18 \%)$ \\
\hline \multicolumn{4}{|l|}{ Limb involvement } \\
\hline Forelimb (39) & 25 & 8 & 6 \\
\hline Hind limb (67) & 33 & 21 & 13 \\
\hline \multicolumn{4}{|l|}{ Location of injury } \\
\hline Proximal (10) & 4 & 5 & 1 \\
\hline Middle (25) & 14 & 5 & 6 \\
\hline Distal (37) & 20 & 11 & 6 \\
\hline Pastern (34) & 20 & 8 & 6 \\
\hline \multicolumn{4}{|l|}{ Tendon sheath involvement } \\
\hline Yes (61) & 32 & 18 & 11 \\
\hline No (45) & 26 & 11 & 8 \\
\hline \multicolumn{4}{|c|}{ No. of structures involved (partially or totally) } \\
\hline $1(48)$ & 36 & 9 & 3 \\
\hline $2(45)$ & 18 & 13 & 14 \\
\hline $3(13)$ & 4 & 7 & 2 \\
\hline \multicolumn{4}{|l|}{ Group } \\
\hline $1(60)$ & 39 & 15 & 6 \\
\hline $2(46)$ & 19 & 14 & 13 \\
\hline \multicolumn{4}{|l|}{ Suturing tendon ends* } \\
\hline Yes (58) & 29 & 16 & 13 \\
\hline No (46) & 29 & 13 & 4 \\
\hline \multicolumn{4}{|l|}{ Lower limb cast* } \\
\hline Yes (84) & 44 & 26 & 14 \\
\hline No (20) & 14 & 3 & 3 \\
\hline
\end{tabular}

*In 2 cases, suturing or cast application was not recorded.

Legend for groups and location of injury: see Table 1.

and 16 horses postoperatively because of persistent lameness, poor prognosis, or persistent infection of the digital flexor tendon sheath]).

Of the 58 horses returning to an equal level of performance, $23(40 \%)$ were used for recreation whereas 35 $(60 \%)$ were competition horses (mainly jumping and dressage horses, trotters, and gallopers). Of the 35 competition horses, $25(71 \%)$ were in group 1 and $10(29 \%)$ in group 2 with, respectively, $23(66 \%), 9(26 \%)$, and $3(8 \%)$ having 1 , 2 , or 3 tendons or ligaments partially or totally transected.

A cast was used in $41(68 \%)$ group 1 horses and 25 $(61 \%)$ returned to equal performance. Nineteen were not cast and $14(77 \%)$ returned to equal performance. In group 2 , a cast was used in all but 1 horse; 19 (44\%) returned to equal performance. The horse treated without a cast returned to a lower level of work.

\section{Influential Outcome Variables (Table 3)}

There was no significant effect of age $(P=.36)$, sex $(P=.33)$, breed $(P=.80)$, or referral clinic $(P=.48)$ on outcome. Because of the difficulty in obtaining an accurate estimation of the time of injury, the effect of the interval between injury and repair could not be evaluated.

With univariate analysis, there was a significantly higher odds ratio (OR) for an unsuccessful outcome when
Table 3 Results of Univariate and Multivariate Analysis for Associations between Clinical Variables and Outcome (Defined as Odds of an Unsuccessful Outcome) after Treatment of 106 Horses with Lacerations of Digital Flexor Tendons, Suspensory Ligament, and/or Distal Sesamoidean Ligaments

\begin{tabular}{|c|c|c|c|c|}
\hline \multirow[b]{3}{*}{ Variable } & \multicolumn{4}{|c|}{ Outcome } \\
\hline & \multicolumn{2}{|c|}{ Univariate Analysis } & \multicolumn{2}{|c|}{ Multivariate Analysis } \\
\hline & $P$-Value & Odds & $P$-Value & Odds \\
\hline Limb involvement (hind, fore) & .14 & 1.84 & - & - \\
\hline Location of injury* & .78 & & & \\
\hline Proximal versus pastern & .30 & 2.14 & & \\
\hline Middle versus pastern & .83 & 1.12 & & \\
\hline Distal versus pastern & .69 & 1.21 & & \\
\hline Tendon sheath involvement & .59 & 0.80 & - & - \\
\hline Groups (2 versus 1 ) & .02 & 2.64 & & \\
\hline No. of structures involved & $<.01$ & & $<.01$ & \\
\hline 1 versus 3 & $<.01$ & 0.15 & $<.01$ & 0.15 \\
\hline 2 versus 3 & .55 & 0.66 & .55 & 0.66 \\
\hline 2 versus 1 & $<.01$ & 4.50 & $<.01$ & 4.5 \\
\hline Suturing tendon & .19 & 1.70 & - & - \\
\hline Cast immobilization & .16 & 2.12 & - & - \\
\hline Duration of casting & .07 & 1.15 & - & - \\
\hline
\end{tabular}

*point estimate and 95\% confidence interval.

Outcome was dichotomized as "successful" when an equal performance was obtained and "unsuccessful" when a lower performance was present or the horse was euthanatized.

Legend for groups and location of injury: see Table 1.

horses belonged to group $2(P=.02, \mathrm{OR}=2.64)$ and when multiple tendons or ligaments were affected compared with only 1 structure $(P<.01)$. Although not statistically significant, there was a tendency $(P=.07)$ for an increased risk of unsuccessful outcome with an increased cast time. Because of the high correlation between the variables "group" and "number of structures affected" (0.61), the least significant one (group, $P=.02$ ) was excluded from further analysis. In the multivariate model, the factor that most significantly affected outcome after surgery was the number of tendons or ligaments injured $(P<.01)$.

\section{Complications}

The most frequent postsurgical complication was thickening of the tendons, subcutaneous tissues, and skin at the level of the initial injury $(n=24)$. This complication occurred irrespective of treatment, occurring in $22 \%$ of horses treated by tenorrhaphy and cast, $30 \%$ treated without tenorrhaphy but with a cast, and $20 \%$ where neither a cast nor tenorrhaphy was used. However, this complication was of low clinical importance because 17 of these 24 horses $(71 \%)$ returned to the previous level of work.

The 2nd most frequent complication was permanent hyperextension of the MCP or MTP joint, which occurred in 15 of 95 horses $(16 \%)$, and information was not available for 11 horses. Six horses had toe elevation requiring permanent trailer shoes and 1 needed surgical arthrodesis of the MTP joint because of DDFT rupture. Temporary fetlock hyperextension (present for $<6$ months) was commonly seen during rehabilitation in horses with complete 
lacerations. Permanent hyperextension of the MCP or the MTP joint (present for $>6$ months) occurred more often in horses with total transection of the SDFT, DDFT, SL, and/or DSL (group 2; 14 of 15 horses) compared with horses with partial lacerations (group 1; 1 horse; Table 1). This complication was also more prevalent in horses with an increasing number of partially or totally transected tendons or ligaments $(1[2 \%], 8$ [21\%], and 6 [50\%] horses with partial or total laceration of 1,2 , and 3 tendons or ligaments, respectively). Of the 15 horses with permanent hyperextension of the MCP or the MTP joint, $14(93 \%)$ returned to a lower level of performance and only $1(7 \%)$ returned to the previous level of work (horse with a total transection of SDFT and DDFT). In contrast, 57 of 80 horses $(71 \%)$ that did not develop this complication returned to the same performance level, whereas $23(29 \%)$ returned to a lower level.

The occurrence of MCP or MTP joint hyperextension was significantly associated with an unsuccessful outcome $(P<.01, \mathrm{OR}=34.70)$. When analyzing the factors associated with the occurrence of this complication, univariate analysis showed a significantly higher odds $(P=.02$, $\mathrm{OR}=9$ ) of developing permanent fetlock hyperextension for a proximal tendon injury compared with a pastern location. On the other hand, suturing the tendon significantly decreased the odds $(P=.02, \mathrm{OR}=0.14)$ of $\mathrm{MCP}$ or MTP joint hyperextension. Cast duration also had a significant influence on the occurrence of fetlock hyperextension $(P<.01)$, as well as group $(P=.002)$ and number of structures affected $(P=.004)$. Because of the high correlation between these last 2 variables, the least significant one (number of structures affected) was excluded from further analysis. In the multivariate analysis, the most significant factor determining the occurrence of fetlock hyperextension was group $(P<.01)$. Group 2 horses (complete transections) were 27.48 times more likely to develop this complication compared with group 1 horses. Duration of casting was also retained in the multivariate model and the odds of developing this complication increased 1.4 times for every week of casting $(P=.01)$.

Several other less frequent postsurgical complications were recorded: 12 horses developed deep cast sores (with casting duration ranging from 2 to 12 weeks). Four returned to the previous level of work, 4 performed at a lower level, and 4 were euthanatized. Wound dehiscence or breakdown was recorded in 7 horses, 3 of which were complicated with necrosis of the tendon. Persistent infectious tenosynovitis of the DFTS was observed in 8 horses $(13 \%$ of the 61 horses with the initial involvement of a tendon sheath). Two horses had a successful outcome after 1 or 2 additional tenoscopic lavages and intrasynovial antibiotic therapy. The other 6 horses were euthanatized: 3 for septic tenosynovitis, 3 because of a poor prognosis or because of owner concerns about the possibilities of recovery.

One horse had an open luxation of the proximal interphalangeal joint after rupture of the SDFT and DDFT (initial lesion with total transection of the SDFT and DDFT at the level of the mid metacarpus). Another horse was reported to have a decreased degree of fetlock joint flexion. Contralateral lameness was observed in 1 horse and a bone sequestrum in 1 horse. Spontaneous ankylosis of the MCP joint occurred in 1 horse that had an initial injury with complete transection of the SDFT and involvement of the fetlock joint.

\section{DISCUSSION}

Although lacerations of the flexor tendons, SL, and/or DSL in the distal aspect of the limb are relatively common, studies determining the outcome and influence of clinical variables are limited and involve relatively low case numbers $\left(n=22,{ }^{4} n=50^{5}\right)$. Because of the diversity of injury types, a large number of cases are required before factors with a significant influence on outcome can be determined. We are unaware of multivariate analysis of a large number of horses with lacerations of the SDFT, DDFT, SL, and/or DSL. The disadvantages of a retrospective multicenter study include differences in patient population and the treatment approaches, and the involvement of many surgeons. This 4-center retrospective study covered 5 years, and allowed us to accumulate 106 horses with lacerations of the SDFT, DDFT, SL, and/or DSL with comparable evaluation of the initial laceration and final outcome.

We found that $82 \%$ of horses with surgically treated flexor tendon and ligament lacerations survived $>1$ year after initial injury, with 55\% returning to an equal level of performance and $27 \%$ to a lower level of performance. These outcomes are similar to those obtained in a study of 50 flexor tendon lacerations where $78 \%$ of the horses survived, $54 \%$ returned to equal performance, and $24 \%$ to a lower level of performance. ${ }^{5}$ Study populations were also comparable for signalment, although the mean age of horses was higher in our study (median, 7 years compared with 4.5 years). In contrast to that study, we chose not to subdivide the "lower performance" category into a group of horses sound enough to be ridden but not at their original level and another group used for breeding or retired to pasture. In our opinion, the postoperative use of a horse for riding, breeding, or pasture was as much dependent on the owners' personal choice as the horse's intrinsic capacity to be ridden. On the other hand, when evaluating the variables with a significant influence on outcome, only horses that were used at their original or intended level were considered "successful" in our study.

Based on multivariate analysis results, the odds for returning to an equal level of performance were mainly dependent on the number of tendons or ligaments affected, with significantly higher odds of an unsuccessful outcome when 2 or 3 structures were affected compared with only 1 structure involved. The greater the number of structures transected, the less support to the fetlock and the other distal joints and thus the greater the likelihood of damage to vessels and nerves. ${ }^{2}$ Although logical, this association between the outcome and the number of structures partially or totally transected has, to our knowledge, not been 
statistically determined previously, nor has involvement of the SL been considered in determining the outcome of flexor tendon lacerations. ${ }^{4,5}$ Moreover, in 1 of these studies $^{4}$ lacerations involving both flexor tendons appeared to have a more favorable prognosis than those involving the SDFT or the DDFT only.

In previous reports, ${ }^{5}$ the prognosis for returning to the previous level of work is poorer in horses with complete tendon or ligament transections compared with horses that have only partial lacerations. Although this was not the most significant factor influencing the return to the previous level of work, group (partial versus total lacerations) certainly also played an important role in the final outcome because of its high correlation with the number of structures affected. Moreover, "group" was the variable with the highest influence on the development of fetlock hyperextension $(\mathrm{OR}=27.24, P<.01)$. Complete transection of a tendon or a ligament may lead to larger gap formation between the lacerated tendon ends, resulting in changes in limb conformation and predisposition to the development of hyperextension of the MCP or the MTP joint.

We found that the limb affected, proximodistal location of the injury, tendon sheath involvement, and method of treatment did not significantly influence the outcome. Although some authors have suggested a better outcome for hind limb flexor tendon and ligament lacerations compared with forelimb lacerations because of normal weight distribution in horses, ${ }^{4}$ we, in agreement with Taylor et al, ${ }^{5}$ could not confirm this. In contrast, the odds for an unsuccessful outcome in our study was 1.84 times higher (not significant) for hind limb injury compared with forelimb injury.

Generally, the involvement of the DFTS in distal flexor tendon lacerations is considered to warrant an unfavorable prognosis, because of impaired tendon healing within the sheath and the possibility of subsequent development of intrasynovial adhesions or septic tenosynovitis. ${ }^{1,4,5}$ Intrasynovial healing is markedly diminished by gap formation, tendon sheath damage, and inadequate immobilization. ${ }^{7}$ We found no effect of injury within the tendon sheath on outcome: 61 of 106 horses had tendon sheath involvement and $50(82 \%)$ survived, with $32(52 \%)$ returning to an equal level of performance. The other 11 $(18 \%)$ were euthanatized but only 3 because of persistent infectious tenosynovitis. This suggests that in most instances, early and aggressive treatment can prevent the development of persistent infectious synovitis and also that repair of a laceration within the tendon sheath is possible without restrictive adhesion formation.

It is generally accepted that the optimal treatment for complete flexor tendon lacerations includes debridement, suturing of tendon ends (provided they can be apposed easily), wound closure, and cast immobilization for at least 6 weeks. ${ }^{2,3}$ It has been proposed that partial lacerations involving $<50-75 \%$ of the tendon need only local debridement, whereas more extensive partial disruptions should be sutured to prevent conversion to complete tears or the development of longitudinal splits between loaded and unloaded parts of the tendon. ${ }^{2,3}$ Consequently, in our horses, most $(60 \%)$ partial lacerations (group 1) were not sutured, whereas most $(77 \%)$ total lacerations (group 2) were sutured. This probably explains why we found no significant effect of suturing on outcome. Experimental studies investigating methods of flexor tendon repair have also demonstrated that cast immobilization without suturing produced a significantly weaker repair and the development of a hyperextended MCP joint. ${ }^{8,9}$ We also found a significantly higher odds for the development of MCP or MTP joint hyperextension when tendons were not sutured $(P=.02)$. In our opinion, suturing may still be beneficial in extensive partial lacerations, providing the edges can be apposed easily.

Although not clinically significant, the most frequent complication was thickening of the tendons, subcutaneous tissues, and skin at the level of the initial injury. It has been suggested that the use of tenorrhaphy in conjunction with external immobilization could minimize scar tissue formation. ${ }^{4}$ We found that $22 \%$ of horses developed hypertrophic scar and tendon thickening at the level of the laceration, and yet $71 \%$ of these horses returned to the previous level of work. Tenorrhaphy was performed in $50 \%$ of these horses and a cast was used in $87 \%$ (this included all horses where the tendons were sutured).

In flexor tendons, at least 6 weeks are required for the tendon to gain sufficient strength to support $450 \mathrm{~kg} .{ }^{2}$ This corresponds to the mean period of cast immobilization in our horses. For complete flexor tendon lacerations in the hind limbs, a full-limb cast is theoretically required to immobilize the forces of the reciprocal apparatus. ${ }^{3}$ Nevertheless, we chose not to use a full-limb cast because of concerns about complications like rupture of the peroneus tertius, ${ }^{10}$ femoral fracture, or coxofemoral luxation. ${ }^{11}$ The use of a half-limb cast for flexor tendon lacerations in the hind limb is mainly to support the MTP joint ${ }^{12}$ but may actually encourage the disruption of a sutured hind limb flexor tendon repair during recovery from general anesthesia because of immobilization of the distal limb only (personal observation, unpublished data). It was not possible to compare casting with no casting for these hind limb lacerations because most (98\%) of the cases were cast.

Although not statistically significant, there was a tendency toward an increased risk of unsuccessful outcome with an increased casting duration. The same variable also had a significant influence on the occurrence of fetlock hyperextension increasing the odds of developing this complication 1.4 times for every week of casting. The tendency for an increased risk of unsuccessful outcome with longer casting periods is likely because horses with more severe lesions were also cast for longer periods. The same explanation could be given for the significant relationship between the duration of casting and the development of permanent fetlock hyperextension. Development of pressure sores is a frequent complication when dealing with distal limb casts in horses (reported prevalence of $80 \%$ for superficial sores and $34 \%$ for deep dermal sores). ${ }^{13}$ Although a much lower incidence occurred in our horses (11\%), this finding should be interpreted with caution because recording might not be 
as accurate as in other studies ${ }^{13}$ that focused on development of cast sores. Nevertheless, their occurrence was not of major clinical consequence because it did not prevent horses from returning to their previous level of work.

In summary, we found that in horses with lacerations of SDFT, DDFT, SL, and/or DSL, there was a high survival rate $(82 \%)$ but only $55 \%$ of the horses returned to their previous level of work. The number of structures affected, either partially or totally, was the major factor determining the odds of returning to an equal level of performance. Permanent MCP or MTP joint hyperextension was the most important postsurgical complication and was significantly more common in horses with complete tendon lacerations.

\section{ACKNOWLEDGMENTS}

We thank Dr. Michael Schramme for initiating this study, the colleagues of the referring clinics, and the referring veterinarians and owners for all their assistance and cooperation in collecting the data.

\section{REFERENCES}

1. Belknap JK, Baxter GM, Nickels FA: Extensor tendon laceration in horses: 50 cases (1982-1988). J Am Vet Med Assoc 1993;203:428-431

2. Bertone AL: Tendon lacerations. Vet Clin North Am Equine Pract 1995;11:293-314

3. Davis CS, Smith RKW: Diagnosis and management of tendon and ligament disorders, in Auer JA, Stick JA (eds): Equine surgery (ed 3). Philadelphia, PA, Saunders, 2006, pp 1098-1109
4. Foland JW, Trotter GW, Stashak TS, et al: Traumatic injuries involving tendons of the distal limbs in horses: a retrospective study of 55 cases. Equine Vet J 1991; 23:422-425

5. Taylor DS, Pascoe JR, Meagher DM, et al: Digital flexor tendon lacerations in horses: 50 cases (1975-1990). J Am Vet Med Assoc 1995;206:342-346

6. Dohoo I, Martin W, Stryhn H: Logistic regression (model building), in Dohoo I, Martin W, Stryhn H (eds): Veterinary epidemiologic research (ed 2). Charlottetown, PEI, AVC Inc., 2003, pp 395-426

7. Jann HJ, Blaik M, Emerson R, et al: Healing characteristics of deep digital flexor tenorrhaphy within the digital sheath of horses. Vet Surg 2003;32:421-430

8. Bertone AL, Stashak TS, Smith FW, et al: A comparison of repair methods for gap healing in equine flexor tendon. Vet Surg 1990;19:254-265

9. Jann HW, Good JK, Morgan SJ, et al: Healing of transected equine superficial digital flexor tendons with and without tenorrhaphy. Vet Surg 1992;21:40-46

10. Sullins KE: Lameness, the tarsus, in Stashak TS (ed): Adams' lameness in horses (ed 5). Philadelphia, PA, Lippincott, Williams \& Wilkins, 2002, pp 931-987

11. Richardson DW: Femur and pelvis, in Auer JA, Stick JA (eds): Equine surgery (ed 3). Philadelphia, PA, Saunders, 2006, pp 1334-1341

12. Schneider RK, Ratzlaff MC, White KK, et al: Effect of three types of half-limb cast on in vitro bone strain recorded from the third metacarpal bone and proximal phalanx in equine cadaver limbs. Am J Vet Res 1998;59:1188-1193

13. Levet T, Martens A, Devisscher L, et al: Distal limb cast sores in horses: risk factors and early detection using thermography. Equine Vet $J$ 2009;41:18-23 